\title{
Selecting stable extragalactic compact radio sources from the permanent astrogeodetic VLBI program ${ }^{\star}$
}

\author{
M. Feissel-Vernier ${ }^{\star \star}$ \\ Observatoire de Paris/CNRS UMR8630, 61 Av. de l'Observatoire, 75014 Paris, France \\ Institut Géographique National/LAREG \\ Received 24 January 2003 / Accepted 25 February 2003

\begin{abstract}
A set of stable compact radio sources is proposed, based on the analysis of VLBI-derived time series of right ascensions and declinations from mid-1989 through May 2002. Five selection schemes are tested, that are based on the usual and Allan standard deviations and on apparent drifts. The efficiency of the selection schemes is characterized by the ability of the sources to support the maintenance of the direction of the axes of the celestial reference frame that they materialize. When compared with the current set of ICRF defining sources, the best performing selection scheme keeps 199 sources and lowers the medium-term frame instability from 28 to 6 microarcsec.
\end{abstract}

Key words. quasars: general - astrometry - reference systems

\section{Introduction}

The permanent astrogeodetic VLBI observing program started in the $1980 \mathrm{~s}$ as a joint effort of several institutions, sponsored mainly by the US National Aeronautical and Space Administration (NASA) and National Oceanic and Atmospheric Administration (NOAA). After undergoing development and international extension, it is now managed by the IVS (International VLBI Service for Geodesy and Astrometry). Astrogeodetic VLBI is currently the only available technique to monitor the irregular Earth's rotation at the required level of accuracy. It will stay so in the foreseable future, as no competing technique is proposed. This program provides the basis for the monitoring of the IAU-recommended International Celestial Reference Frame (ICRF, Ma et al. 1998; see also IERS 1999 for the ICRF-Ext.1) to which other celestial reference frames, e.g. the Hipparcos catalogue, are referred.

At the end of 2002, over three million observations of more than 700 compact extragalactic radio sources have been accumulated. This data set includes extremely uneven observation rates. However, about half of the objects are well enough monitored so that the medium and long term stability of their directions can be investigated.

The observed objects are selected by the IVS among quasars and galaxy nuclei that are compact when observed in the $X$-band $(4 \mathrm{~cm})$ and $S$-band $(13 \mathrm{~cm})$ with baseline lengths extending to several thousands kilometers. However, when

^ Table 6 is only available in electronic form at http: //wWw. edpsciences.org

$\star \star$ e-mail: feissel@ensg.ign.fr observed at the current level of precision (a fraction of milliarcsecond, mas), no object is really pointlike. Apparent motions, if existing, may be related to the existence of jets originating in the sources. While the background source structure is assumed to stay fixed, quasi periodical oscillations may exist, as well as apparent drifts that are unlikely to continue indefinitely, but with time characteristics that are not well known.

This observational situation may come into contradiction with the basic assumption that, globally, the set of selected object has a no net rotation relative to the quasi inertial space. However, little is presently known about the actual behaviour of these hundreds of sources, so that the solution to solve this difficulty appears to be the selection of sources that best match the no-motion model, in order to minimize the uncontrolled global rotation. The authors of the ICRF (Ma et al. 1998) set up three classes of sources, using qualitative and quantitative criteria, such as apparent drift, formal uncertainty of the global coordinates over 1980-1995, source stucture index. They used only the sources they considered as the most reliable (the defining sources) for the definition of the ICRF orientation. A second set of apparently reliable sources that had not been well enough observed in 1995 were considered as candidates. Finally, the remaining sources, considered as unstable, were labelled other sources.

In this paper, we study statistical schemes to detect the most stable sources in the available data, that would be used for the maintenance of the ICRF and the monitoring of the Earth's rotation. The study is entirely based on the series of individual source coordinates derived by Fey (2002) over 1979-2002. We extract from this data set the data over 1989.5-2002.4, 


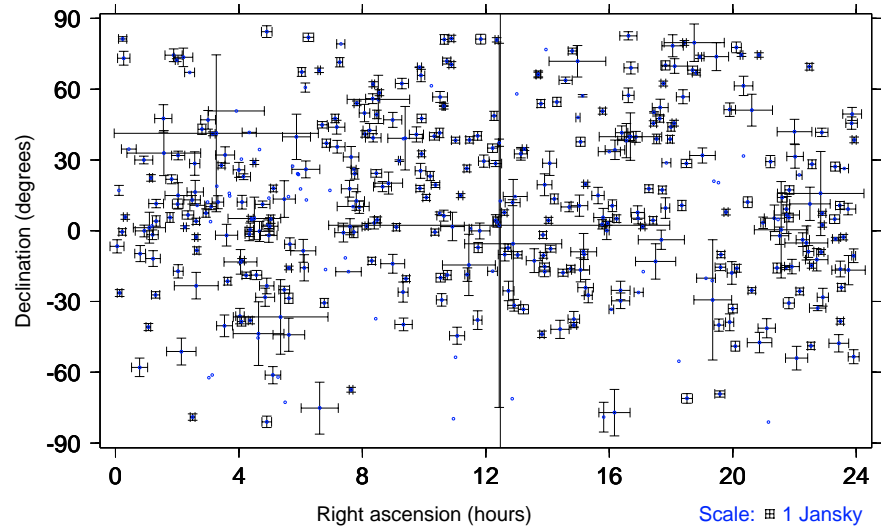

Fig. 1. The most observed ICRF radio sources. Total flux at wavelengths $6 \mathrm{~cm}$ (vertical bars), and $11 \mathrm{~cm}$ or $15 \mathrm{~cm}$ (horizontal bars) are shown when available.

i.e. 3.1 million observations of 707 sources, representing $87 \%$ of the total observations.

\section{The observations}

The observations consist of time series derived from a set of three analyses of the existing VLBI observing sessions up to May 2002. Each analysis results in time series of coordinates per session for one third of the sources ("arc" sources), the coordinates of the other sources being treated as "global", i.e. they are assumed to stay fixed in time and are estimated globally. Each analysis includes a no-net-rotation (NNR) condition with respect to the ICRF based on the ICRF defining sources.

To avoid inconsistencies due to the NNR condition realization in the three analyses, and that reach the level of 50 microarcsec ( $\mu \mathrm{as})$, we consider here for each source the time series of its coordinate differences with their global weighted mean.

Figure 1 gives the sky distribution of the most observed ICRF radio sources. The mean flux at $6 \mathrm{~cm}$ and 11 or $15 \mathrm{~cm}$ is shown when available at the IERS Celestial Reference System Product Center (2002). The observation rates histogram are shown in Fig. 2 for the 707 sources.

If the source structure is extended or not circular, its apparent direction may change as a function of the length and orientation of the baselines. Most of the known activity of quasars takes the form of jets, i.e., aligned emissive structures that cause an apparent motion of the observed phase center relative to a fixed background. Therefore, the source structure often changes with time. In principle it is possible to accurately correct this effect, provided that repeated maps of the sources are available (Charlot \& Sovers 1997).

However, although series of source maps are routinely derived for the northern hemisphere (Fey \& Charlot 1997), the source structure correction is not implemented in the existing astrogeodetic analysis software. As a result, most objects exhibit time variations of their position in some preferred direction.

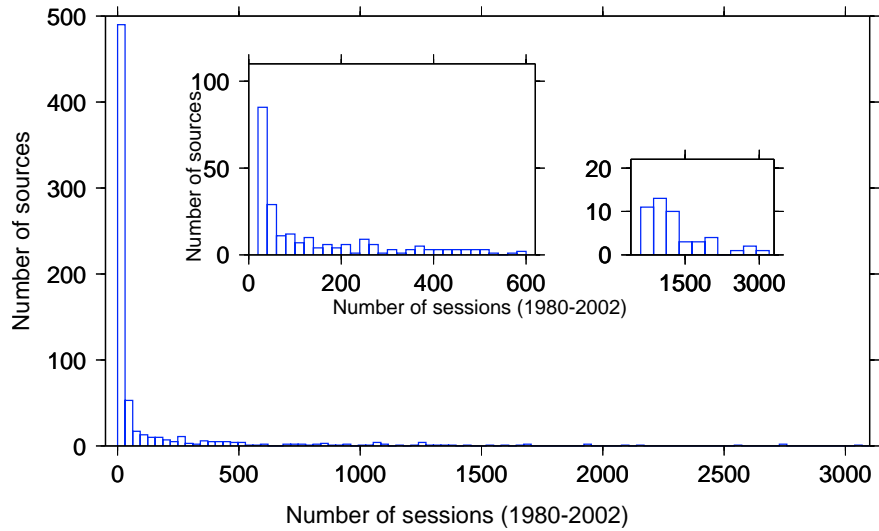

Fig. 2. Observation rates. Number of sessions in which a given source is observed. The leftmost abscissae limit in the right insert is equal to 600 .

\section{Selection schemes}

An earlier study (Gontier et al. 2001) has shown that the improvements in VLBI technology, the development of the observing network and the extension of the set of observed objects that took place in operational VLBI, brought the astrometric results to its current precision towards the end of the first decade. Starting about 1990, individual time series of source coordinates stabilize. Therefore, the selection schemes under study are applied to data after 1989.5. Note that values and plots of yearly averages for sources observed since 1984 are available (IERS 2003).

A set of stable sources is selected in a two-step process, based on series of one-year coordinates, obtained as the mean of the sessions's coordinates weighted according to the corresponding uncertainties:

1. A first selection is made on the basis of continuity criteria for one-year weighted average coordinates.

(a) Length of observation period longer than five years.

(b) Not less than two observations of the source in a given session.

(c) One-year average coordinates based on at least three observations. As this number may be considered as too small, a test was made for comparison with one-year average coordinates based on at least twelve observations.

(d) Not more than three successive years with no observations, conditions (b) and (c) being met.

(e) At least half of the one-year averages available over the source observation span.

This first screening keeps 362 sources for the years centered at 1990.0 through 2002.0. These include 141 defining sources, 130 candidates, 87 other and 4 new sources, i.e. $67 \%$ of the defining sources, $44 \%$ of the candidates and $85 \%$ of the others.

2. The time series of yearly values of $\alpha \cos \delta$ and $\delta$ are then analysed in order to derive

(a) the linear drift (least squares estimation); 
(b) the standard deviation of a single value referred to its global average, and

(c) the Allan standard deviation for a one-year sampling time. The Allan variance of a time series $x_{i}$ with $N$ items and sampling time $\tau$ is defined as:

$$
\sigma_{\mathrm{A}}^{2}(\tau)=\frac{1}{2 N} \sum_{i}\left(x_{i+1}-x_{i}\right)^{2}
$$

The Allan variance analysis (Allan 1966, see a review of these methods in Rutman 1978) allows one to characterize the power spectrum of the variability in time series, for sampling times ranging from the initial interval of the series to $1 / 4$ to $1 / 3$ of the data span, in our case one year through four years. This method allows one to identify white noise (spectral density $S$ independent of frequency $f$ ), flicker noise $\left(S\right.$ proportional to $\left.f^{-1}\right)$, and random walk ( $S$ proportional to $f^{-2}$ ). Note that one can simulate flicker noise in a time series by introducing steps of random amplitudes at random dates. In the case of a white noise spectrum (an implicit hypothesis in the current ICRF computation strategy), accumulating observations with time eventually leads to the stabilisation of the mean position. In the case of flicker noise, extending the time span of observation does not improve the quality of the mean coordinates. A convenient and rigorous way to relate the Allan variance of a signal to its error spectrum is the interpretation of the Allan graph, which gives the changes of the Allan variance for increasing values of the sampling time $\tau$. In logarithmic scales, slopes $-1,0$ and +1 correspond respectively to white noise, flicker noise and random walk noise.

The distributions of the above statistics applied to the 362 preselected sources are shown in Fig. 3. The normalized linear drift is the absolute value of the least-square derived linear drift divided by its formal uncertainty. This unitless number measures the degree of statistical significance of the estimated velocity. Note that the histograms are quite similar for right ascensions and declinations, except for the standard deviations of the one-year average coordinates, where the declinations tend to be noisier than right ascensions. This may be related to analysis difficulties remaining after the sizeable improvement that was brought to the declination determinations by the adoption of the gradient function correction (McMillan \& Ma 1997).

Based on these statistics, rules are set up to derive source stability indices. For each local coordinate $(\alpha \cos \delta$ and $\delta)$, three partial indices are defined, as shown in Table 1. The thresholds are set after the distributions of Fig. 3. The partial stability indices range from 1 (most stable) through 3 (least stable). A rejection value (10) is associated to very large drifts or standard deviations. Given the length of the available time series (up to 13 years), one could consider the Allan standard deviation for sampling times longer than one year (e.g. two or four years). The one-year interval is preferred because its estimation is expected to be more robust than for longer time spans.

The partial stability criteria derived by applying the thresholds of Table 1 are then combined in five different ways, each one representing a different balance in the roles of the standard deviations and the drifts. Fifty sources were found to have
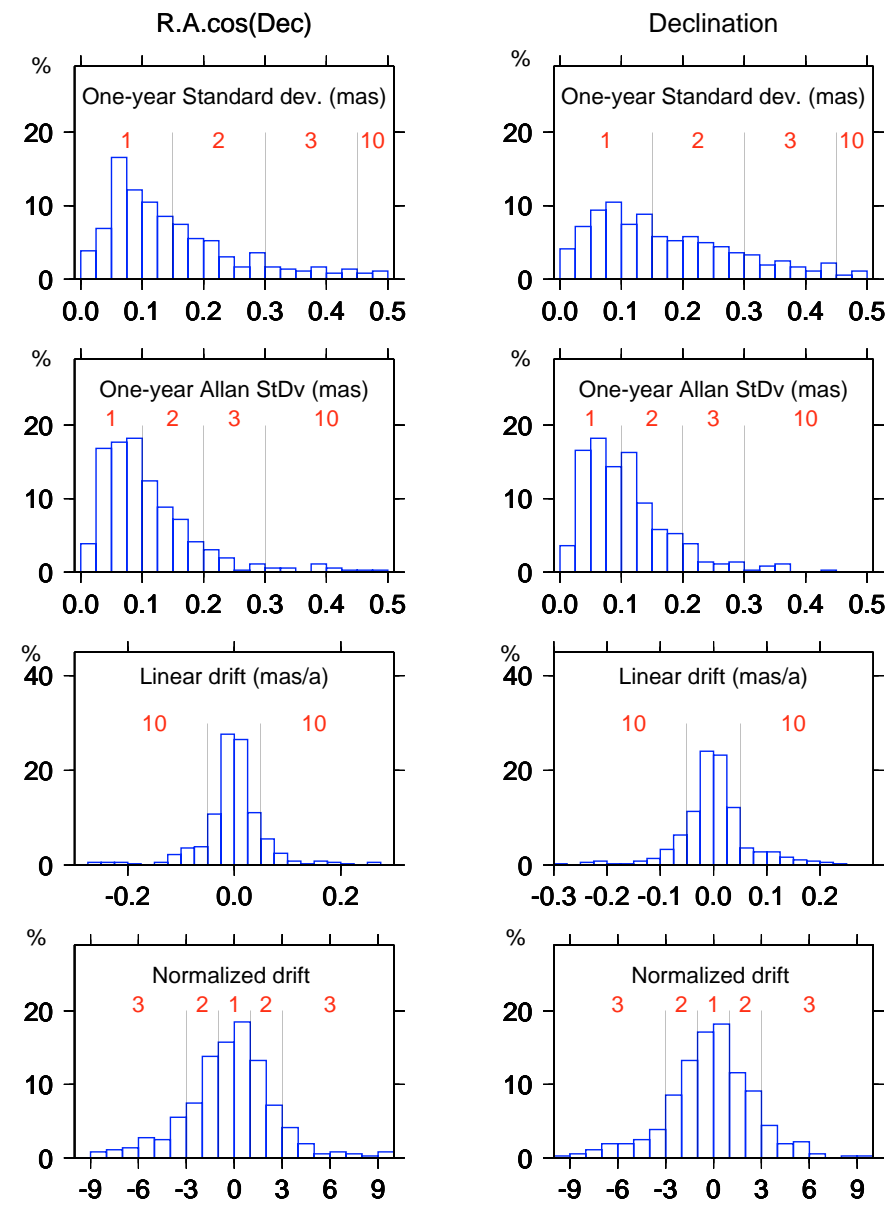

Fig. 3. Distributions of the partial stability criteria for the well observed sources over 1989.5-2002.4. The thresholds and corresponding partial indices listed in Table 1 are shown.

apparent drifts larger than $50 \mu$ as/year. With at least one partial criterium equal to the rejection value (10), they are excluded a priori in the five options.

The partial indices combinations are as follows.

(a) Usual standard deviation only.

(b) Allan standard deviation only.

(c) Normalized velocity only.

(d) Usual standard deviation and normalized velocity.

(e) Allan standard deviation and normalized velocity.

The global stability index is then defined as the average of the partial indices for $\alpha \cos \delta$ and $\delta$, i.e. two values per source in cases (a), (b) and (c) and four values per source in cases (d) and (e). The stable sources are those which get a stablity in$\operatorname{dex} \leq 2.5$.

Combination (a) takes into account the statistical scatter of yearly coordinates, paying no attention to the fact that they belong to a time series. Combination (b) takes this aspect into account in a statistical way, while combination (c) takes it into account in a deterministic way, by modelling an assumed physical phenomenon. Combinations (d) and (e) mix the statistical and deterministic approaches. 
Table 1. Partial stability criteria. The values range from 1 (best) through 3 (worse), with a rejection value of 10 .

\begin{tabular}{|c|c|c|}
\hline Statistics & Threshold & Partial index \\
\hline \multicolumn{3}{|c|}{ Standard deviation (Stdv) } \\
\hline & $\operatorname{Stdv} \leq 150 \mu$ as & 1 \\
\hline & $150 \mu$ as $\leq \operatorname{Stdv} \leq 300 \mu$ as & 2 \\
\hline & $300 \mu$ as $\leq \operatorname{Stdv} \leq 450 \mu$ as & 3 \\
\hline & $\operatorname{Stdv} \geq 450 \mu$ as & 10 \\
\hline \multicolumn{3}{|c|}{ Allan Standard deviation (AlSd) } \\
\hline & $\mathrm{AlSd} \leq 100 \mu$ as & 1 \\
\hline & $100 \mu$ as $\leq \mathrm{AlSd} \leq 200 \mu$ as & 2 \\
\hline & $200 \mu$ as $\leq \mathrm{AlSd} \leq 300 \mu$ as & 3 \\
\hline & $\mathrm{AlSd} \geq 300 \mu$ as & 10 \\
\hline \multicolumn{3}{|c|}{ |Drift| (Vel) } \\
\hline & $\mathrm{Vel} \leq 10 \mu \mathrm{as} /$ year & 1 \\
\hline & $\mathrm{Vel} \geq 50 \mu$ as/year & 10 \\
\hline \multicolumn{3}{|c|}{ |Normalized drift| (Nvl) } \\
\hline & $\mathrm{Nvl} \leq 1$ & 1 \\
\hline & $1 \leq \mathrm{Nvl} \leq 3$ & 2 \\
\hline & $\mathrm{Nvl} \geq 3$ & 3 \\
\hline
\end{tabular}

\section{Testing the source selections}

Out of the 362 preselected sources the investigated stability schemes produce between 186 and 208 stable sources, numbers to be compared to the 212 defining sources in the ICRF. To test their efficiency with respect to the maintenance of the axes of the celestial reference frame that they materialize, we consider the 13 yearly differential reference frames (1990.0-2002.0) that are formed by the set of stable sources observed in each year. The yearly differential rotation angles $A_{1}(y), A_{2}(y), A_{3}(y)$ around the axes of the equatorial coordinate system for year $y$ are then computed for the 13 years. As the time series of yearly coordinates for each source are referred to the source global average ones, the rotation angles are not affected by the discrepancies between the particular realizations of the no-net-rotation condition attached to the three data treatments that produced the original data used in this work.

Table 2 lists efficiency estimates for the five stability schemes and for the ICRF defining sources. These estimates are the usual standard deviation that characterizes the scattering of the sets of rotation angles, and their Allan standard deviations for one-year and four-year sampling times, that characterize their stability in time. Note that if the time series of the rotation angles have white noise, the four-year Allan standard deviation will be equal to half (square root of one fourth in sampling time) the one-year one. In the case of flicker noise, both values will be equal.

The mixed schemes (d) and (e) keep more stable sources than the single-criterion ones, and yet they insure smaller scattering and better stability. Scheme (e), that takes into account the time series aspect of the data, has slightly better performances that scheme (d), with 5\% less sources. As an example, source $2145+067$, which shows an abrupt change of coordinates ( -0.5 mas from 1998.0 to 1999.0$)$ in a context of moderate drift ( $22 \mu$ as/year), is detected as unstable only by the Allan standard deviation.
The estimated efficiency of the ICRF defining sources is worse than that of selection (e) by nearly a factor of three for the one-year estimates and a factor of five in the longer term. The time series of the yearly rotation angles estimated on the basis of the defining sources have flicker noise at the level of $28 \mu$ as. The type of noise of those based on the stable sources (e) is closer to white noise.

\section{Discussion}

Table 6 (only available in the electronic version of the paper) gives details of the implementation of the selection scheme: number of sessions in which the source was observed, number of yearly points, one-year Allan standard deviation and drift, and four different stability indices. Note that in this table any stability index value larger than 4 is set to this value. For reference, the tables reproduces the values of the source redshifts and fluxes available at the IERS Celestial Reference System Product Center (IERS 2002). The stability indices are the following ones.

1. The ICRF source status: $d=$ defining, $\mathrm{c}=$ candidate, $\mathrm{o}=$ other, $\mathrm{n}=$ not available in ICRF Ext-1.

2. The source structure index, when available in ICRF-Ext.1. This index (Fey \& Charlot 2000) qualifies the level of position disturbance expected as a result of the the source structure ( 1 for the least disturbed, 4 for the most disturbed)

3. The stability criterion derived in this paper; values 1 and 2 correspond to stable sources, 3 to unstable, and 4 to highly unstable or drifting. The stability indices are rounded off.

4. The same stability criterion as above, but keeping only yearly averages based on at least twelve observations (instead of three).

When imposing a minimum number of twelve observations in the yearly averages instead of three, the difference in stability estimates are as follows.

- 87 sources less as preselected in the first step, 36 because of criterion (d), and 51 because of criterion (e). Note that $2 / 3$ of these sources are detected as highly unstable or drifting (stability index 4) while most of the reamining ones are detected as highly stable (stability index 1).

- The total number of stable sources drops from 199 to 169.

- The stability diagnoses in the two cases are identical, with very few exceptions.

The source names listed are the 8-character IERS names. The alternate given are those used in the IVS operation. However, most sources have a number of other usual alternate names, the list of which can be found on an ftp site (IERS 2002) or obtained interactively at URL http://hpiers.obspm/fr/icrs-pc/icrf/srcform.html.

Table 3 shows the distribution of the type of noise for the stable and unstable sources as qualified by scheme (e). Sources with white noise are the most frequent globally. They are roughly equally distributed in the stable and unstable sources, as are the less frequent flicker noise sources. The sources with random walk noise are quite few, in particular among the stable 
Table 2. Efficiency estimates for the tested stability schemes.

\begin{tabular}{lccrrr}
\hline \hline $\begin{array}{c}\text { Source } \\
\text { selection } \\
\text { scheme }\end{array}$ & $\begin{array}{c}\text { Nb. of } \\
\text { sources } \\
\text { kept }\end{array}$ & $\begin{array}{r}\text { Std } \\
\text { dev. } \\
\mu \text { as }\end{array}$ & $\begin{array}{r}\text { Allan Std dev. } \\
\text { 1 year }\end{array}$ & $\begin{array}{r}\text { 4 years } \\
\mu \text { as }\end{array}$ \\
\hline (a) & Stdv & 195 & 11.5 & 10.1 & 8.7 \\
(b) & AlSd & 186 & 14.1 & 8.8 & 8.9 \\
(c) & Nvl & 192 & 11.3 & 9.6 & 5.2 \\
(d) & Stdv+Nvl & 208 & 11.3 & 9.8 & 6.7 \\
(e) & AlSd+Nvl & 199 & 10.8 & 9.4 & 5.9 \\
ICRF Defining & 212 & 25.6 & 26.0 & 27.6 \\
\hline
\end{tabular}

Table 3. Type of noise for the stable and unstable sources as qualified by scheme (e), expressed as the percentage of the types of noise in both categories.

\begin{tabular}{lrrrr}
\hline \hline Type & \multicolumn{2}{c}{ Stable sources } & \multicolumn{2}{c}{ Unst. sources } \\
of noise & RA & Dec & RA & Dec \\
\hline White & $56 \%$ & $69 \%$ & $55 \%$ & $60 \%$ \\
Flicker & $40 \%$ & $30 \%$ & $38 \%$ & $32 \%$ \\
Random walk & $4 \%$ & $1 \%$ & $7 \%$ & $8 \%$ \\
\hline
\end{tabular}

Table 4. Stable sources with random walk noise. The table gives the source stability index, the slope of the Allan variance graph and the Allan standard deviation for a four-year sampling time.

\begin{tabular}{lccccc}
\hline \hline \multirow{2}{*}{ Source } & Stb & Slope & $\begin{array}{c}\text { 4-yr AlSd } \\
\mu \text { as }\end{array}$ & Slope & $\begin{array}{c}\text { 4-yr AlSd } \\
\mu \text { as }\end{array}$ \\
\hline & ind & & & \\
$0119+041$ & 2 & $0.8 \pm 0.2$ & 350 & & \\
$0133+476$ & 2 & & & $0.7 \pm 0.2$ & 103 \\
$0229+131$ & 2 & $1.1 \pm 0.1$ & 236 & & \\
$0234+285$ & 2 & $1.1 \pm 0.4$ & 95 & & \\
$0552+398$ & 2 & $0.7 \pm 0.1$ & 199 & & \\
$0919-260$ & 1 & $1.1 \pm 0.1$ & 131 & & \\
$1611+343$ & 2 & & & $0.6 \pm 0.1$ & 273 \\
$2128-123$ & 2 & $1.4 \pm 0.2$ & 615 & & \\
\hline
\end{tabular}

sources. Note that these estimations are based on too short time series (13 values at most) to guarantee their robustness. Taking into account the type of noise may be considered for further improvement of the qualifying scheme, when longer time series become available. Table 4 lists the eight stable sources with random walk noise in either right ascension or in declination, i.e. for which the slopes of the Allan graph are close to +1 . With the exception of 0119+041 and 2128-123, their Allan standard deviation for a four-year sampling time is small enough to deserve a partial stability index of 2 .

Figure 4 shows the relationship of the ICRF categories with the two components of the source stability index derived in this study for the 312 well observed sources with apparent drifts smaller than $50 \mu$ as/year over 1989.5-2002.4. The graphs give the positions of the defining, candidate and other sources as a function of both the one-year Allan standard deviation and the normalized drift. Although a number of defining sources fall in the lower-left zone which correspond to small partial
Table 5. Stability index and ICRF source qualifiers: number of sources.

\begin{tabular}{crrrrrr}
\hline \hline Stab. & \multicolumn{3}{c}{ Source status } & \multicolumn{3}{c}{ Structure index } \\
index & Def. & Can. & Oth. & 1 & 2 & $3-4$ \\
\hline 1 & 33 & 26 & 15 & 46 & 11 & 7 \\
2 & 48 & 42 & 34 & 76 & 26 & 6 \\
3 & 2 & 1 & 4 & 6 & 2 & 0 \\
4 & 58 & 61 & 34 & 70 & 26 & 13 \\
\hline
\end{tabular}
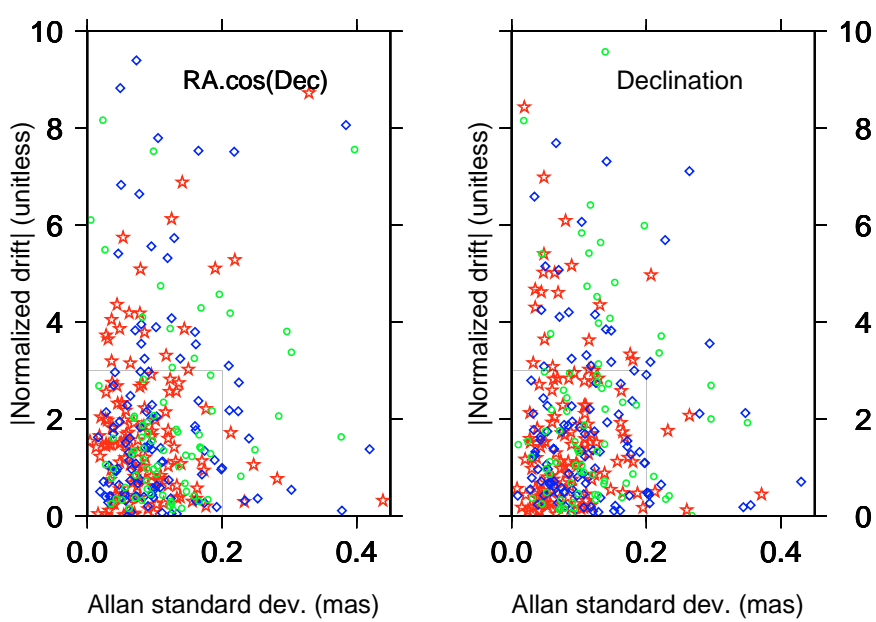

Fig. 4. Partial stability criteria and ICRF source classes: defining (stars), candidate (diamonds) and other (circles). The lower left rectangle includes the sources selected as stable.

stability indices, some of them are clearly detected as unstable. The candidate sources are globally in the same situation. Conversely, a number of "other" sources, i.e. considered as having questionable stability in the ICRF work, are detected as stable. Compared to the ICRF selection, the proposed scheme rescues a number of sources that are in fact efficient for maintaining the ICRF axes directions.

Table 5 gives the relationship of the global stability index with two ICRF qualifiers: the source status (defining/candidate/other) and structure index. Again, there is no particular correlation of the instantaneous structure indices with the time stability ones. This finding is of interest in the context of studying the dynamics of the quasars activity. Similarly, there is no clear relationship of the source total fluxes in the $X$ and $S$ bands with their direction time variability. A possible relationship with the flux variability remains to be investigated.

When compared to the current ICRF defining sources, the selection scheme developed in this study achieves improved time stability of yearly reference frames, which implies improved internal consistency. This has consequences not only in astrometric applications, but it may also have bearings in the geophysical interpretation of the Earth's orientation in inertial space. As an example, Dehant et al. (2003) have shown that source instabilities perturb nutation determinations at the level of tens of $\mu$ as, comparable to that of the excitation of the Earth's rotation axis orientation by diurnal oscillations in the atmosphere.

Finally, we recommend that the set of selected stable sources be considered on the one hand in any future 
improvement of the International Celestial Reference Frame, and on the other hand in the scheduling of repeated VLBI sessions for Earth rotation, geodesy and astrometry.

Acknowledgements. The data analysis in this study is entirely based on the time series of coordinates of 707 radio source derived by Alan Fey (USNO) using 3.6 millions of observations accumulated in various US and international programs since 1979.

\section{References}

Allan, D. W. 1966, Proc. IEEE, 54, 221

Charlot, P., \& Sovers, O. J. 1997, 23rd IAU GA, JD 7, August 1997, Kyoto, Japan
Dehant, V., Feissel-Vernier, M., de Viron, O., et al. 2003, JGR, DOI 2002JB001763, in press

Fey, A. L., \& Charlot, P. 1997, ApJS, 111, 95

Fey, A. L., \& Charlot, P. 2000, ApJS, 128, 17

Fey, A. L. 2002, private communication

Gontier, A.-M., Le Bail, K., \& Feissel, M. 2001, A\&A, 375, 661

IERS 1999, ftp://hpiers.obspm.fr/icrs-pc/icrf/iau/ icrf-Ext. 1

IERS 2002, ftp://hpiers.obspm. fr/icrs-pc/icrf/iau/ icrf.car

IERS 2003, http://hpiers.obspm. fr/icrs-pc/icrf/timeseries/liste.html

Ma, C., Arias, E. F., Eubanks, T. M., et al. 1998, AJ, 116, 516

McMillan, D. S., \& Ma, C. 1997, GRL, 24, 453

Rutman, J. 1978, Proc. IEEE, 66, 1048 